\title{
Epidemiology of Ureaplasma urealyticum and Mycoplasma hominis in the semen of male outpatients with reproductive disorders
}

\author{
XIAOFEI ZHU, MIN LI, HUILING CAO, XUEWEN YANG and CHUNBING ZHANG \\ Department of Laboratory Medicine, Jiangsu Province Hospital of Traditional Chinese Medicine \\ Affiliated to Nanjing University of Chinese Medicine, Nanjing, Jiangsu 210029, P.R. China
}

Received January 30, 2015; Accepted March 10, 2016

DOI: $10.3892 /$ etm.2016.3409

\begin{abstract}
The aim of the present study was to investigate the association between Mycoplasma infection and infertility in male outpatients among a Chinese population. Epidemiological data, including prevalence, age distribution and antibiotic resistance profile of patients with an Ureaplasma urealyticum or Mycoplasma hominis infection were collected between 2009 and 2012. Among the 7,374 individuals analyzed, 3,225 patients $(43.7 \%)$ were determined to be positive for infection with U. urealyticum, M. hominis or for both Mycoplasmas. Among the positive cultures, $U$. urealyticum was detected most frequently, while $M$. hominis was rarely found. The age range of 25-34 years was the preferred period for the positive detection. Tetracyclines and josamycin were the most effective agents against both genital Mycoplasmas, including in the case of co-infection. Macrolides (erythromycin, roxithromycin, azithromycin, clarithromycin except for josamycin) were effective against the majority of $U$. urealyticum clinical isolates, but were naturally resisted by $M$. hominis in this study. Fluoroquinolones had the lowest activity against $U$. urealyticum, particularly in cases of $M$. hominis co-infection. Furthermore, fluoroquinolones showed a similar pattern of drug resistance against $M$. hominis to that of $U$. urealyticum. Antibiotic resistance did not vary significantly over the test period. Notably, an elevated multi-drug resistance rate was observed in patients co-infected with both Mycoplasmas. In light of the epidemiological characteristics of genital Mycoplasmas in male infertility patients, the present results may aid Chinese clinicians to implement rational drug usage and avoid the overuse of antibiotics.
\end{abstract}

Correspondence to: Professor Chunbing Zhang or Professor Xuewen Yang, Department of Laboratory Medicine, Jiangsu Province Hospital of Traditional Chinese Medicine Affiliated to Nanjing University of Chinese Medicine, 155 Hanzhong Road, Nanjing, Jiangsu 210029, P.R. China

E-mail: nju_tcm@163.com

E-mail: firstfisher@163.com

Key words: antimicrobial susceptibility, Ureaplasma urealyticum, Mycoplasma hominis, semen, infertility

\section{Introduction}

Mycoplasmas belong to the class Mollicutes which also contains Ureaplasmas, Acholeplasmas, Spiroplasmas and the newly classified Haemoplasmas (1). Mycoplasmas are characterized by a small size, lack of cell wall, extremely fastidious in vitro environmental requirements and a tendency to form centered colonies on solid media (2).

$\beta$-lactam antibiotics and vancomycin are inactive against Mycoplasmas, as their target is the cell wall (3). Mycoplasma and Ureaplasma spp. are currently susceptible to agents that interfere with protein synthesis, including tetracycline, macrolides, aminoglycosides and chloramphenicol, and the fluoroquinolones that inhibit topoisomerases $(3,4)$. However, resistance to these agents is increasing, as a consequence of the spread of the tet $M$ gene which confers resistance to tetracycline (5-7). Clindamycin, fluoroquinolones or other macrolides may be used following the failure of therapy with tetracycline or erythromycin (8).

The extent of bacterial resistance varies geographically, depending on the use of different antibiotics and the history of previous antimicrobial exposure among various populations (9). To date, with regard to the rising infertility in China, an elevated incidence of genital Mycoplasma infection in female outpatients was identified by Wang et al (10); however, there is currently no data regarding the infection prevalence of genital Mycoplasmas in the male partners of infertile couples. For these reasons, it is important to implement surveillance studies on the prevalence and antimicrobial susceptibilities of these species in the Chinese male population. The purposes of this study were as follows: i) Analyze the prevalence of urogenital mycoplasma; ii) investigate the susceptibilities of a large number of clinical isolates of M. hominis and $U$. urealyticum to various antibiotics; and iii) compare changes in the antibiotic susceptibilities of these microorganisms between 2009 and 2012 .

\section{Materials and methods}

Patients. Between January 1st, 2009 and December 31st, 2012 , a total of 7,374 male outpatients with suspected reproductive disorders were enrolled from the Affiliated Hospital of Nanjing University of Chinese Medicine (Nanjing, China), and underwent an inspection of the reproductive system. The patients 
Table I. Distribution of Ureaplasma urealyticum and Mycoplasma hominis (single- and co-infection) among male patients in different age groups during the study period.

\begin{tabular}{lccrr}
\hline $\begin{array}{l}\text { Age group } \\
\text { (years) }\end{array}$ & M. hominis & U. urealyticum & \multicolumn{1}{c}{$\begin{array}{l}\text { M.hominis }+ \\
\text { U. urealyticum }\end{array}$} \\
\hline $18-24$ & $2(0.0)$ & $445(14.1)$ & $8(10.8)$ & $453(100)$ \\
$25-29$ & $17(100.0)$ & $1,047(33.3)$ & $23(31.1)$ & $1,073(100)$ \\
$30-34$ & $9(0.0)$ & $1,128(35.8)$ & $31(41.9)$ & $7(9.5)$ \\
$35-39$ & $1(0.0)$ & $476(15.1)$ & $5(6.8)$ & $57(100)$ \\
$\geq 40$ & $0(0.0)$ & $52(1.7)$ & $74(100)$ & $3,225(100)$ \\
Total & $29(100.0)$ & $3,148(100)$ &
\end{tabular}

Results are presented as $\mathrm{n}(\%)$.

ages ranged between 18 and 47 years. The present study was approved by the ethical committee of the Nanjing University of Chinese Medicine.

Specimens, culture and antimicrobial susceptibility testing. All semen samples were obtained by masturbation and inoculated into the Mycoplasma Susceptibility kit (cat. no. 20140317119877; Autobio Diagnostics Co.,Ltd.,Zhengzhou, China) within $1 \mathrm{~h}$, according to the manufacturer's guidelines. The microbiological principle used by the Mycoplasma identification verification and antibiotic susceptibility testing kits was as follows: During growth, U. urealyticum and M.hominis metabolize urea and arginine, respectively, which changes the color of the culture medium (e.g., from yellow to red). Susceptibility results were obtained at two concentrations for 12 antibiotics: Erythromycin, roxithromycin, josamycin, tetracycline, doxycycline, minocycline, levofloxacin, ofloxacin, azithromycin, clarithromycin, ciprofloxacin and sparfloxacin (Thermo Fisher Scientific Oxoid, Ltd., Basingstoke, UK). The susceptibility of the bacteria to each antibiotic was graded as either 'susceptible', 'intermediate' or 'resistant' (11). Bacterial growth was evaluated following a two-day incubation period at $37^{\circ} \mathrm{C}$. The results were interpreted as follows: Negative result was clear and a color change of $>10^{4}$ units was considered to indicate infection. Clinical and Laboratory Standards Institute guidelines were used to categorize the results for bacterial susceptibility or resistance to antibiotics (12).

Statistical analysis. SPSS software, version 12.0 (SPSS, Inc., Chicago, IL, USA) was used to conduct the data analysis. The $\chi^{2}$ test was used to compare the occurrence of strains susceptible or resistant to different antibiotics. $\mathrm{P}<0.05$ was considered to indicate a statistically significant difference.

\section{Results}

Prevalence of $U$. urealyticum and M. hominis. Among the 7,374 specimens tested, 3,225 (43.7\%) were positive for genital Mycoplasmas. Of these, 3,122 specimens were positive for U. urealyticum (42.3\%), 29 were positive for M. hominis $(0.4 \%)$ and 74 were positive for both (1.0\%) (Fig. 1). The distribution of $M$. hominis and $U$. urealyticum according to age group is shown in Table I. The prevalence rates of $U$. urealyticum

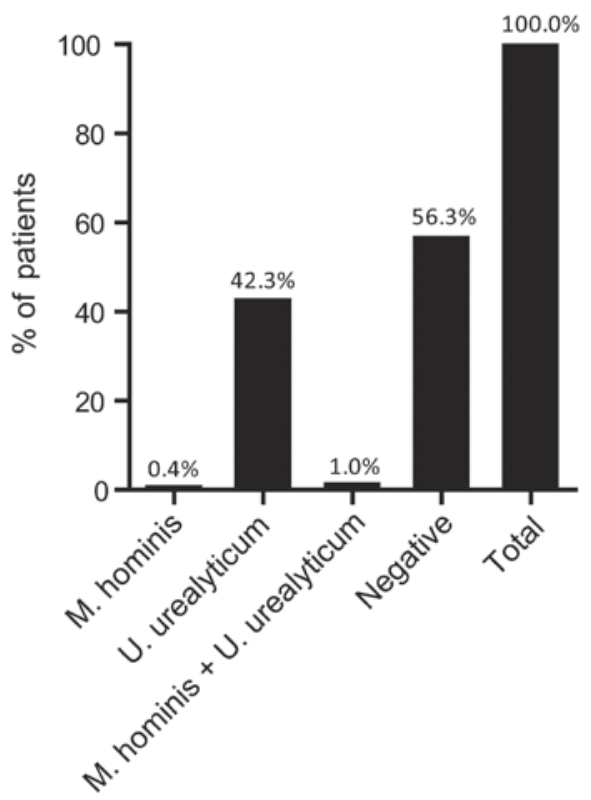

Figure 1. Constituent ratio of $U$. urealyticum, M. hominis and co-infection. U. urealyticum, Ureaplasma urealyticum; M. hominis, Mycoplasma hominis.

and of both Mycoplasmas were highest in patients aged 25-34 years, whereas M. hominis occurred predominantly in patients between the ages of 25 and 29 years.

Antimicrobial susceptibility patterns over the test period. The antimicrobial susceptibilities of M. hominis and $U$. urealyticum are shown in Fig. 2. Tetracyclines (tetracycline, minocycline, doxycycline) were the most active agents against both genital Mycoplasmas even in the case of co-infection. Macrolide (erythromycin, roxithromycin, azithromycin, clarithromycin and josamycin) remained effective against the majority of $U$. urealyticum clinical isolates. However, macrolides, with the exception of josamycin, were naturally resisted by $M$. hominis. Likewise, in the persons infected with both genital Mycoplasmas, the antibacterial activity of macrolides except for josamycin were not significant. Josamycin was the only macrolide observed to be effective against $M$. hominis and co-infection. Fluoroquinolones (ciprofloxacin) had the lowest activity against $U$. urealyticum, particularly in patients with 

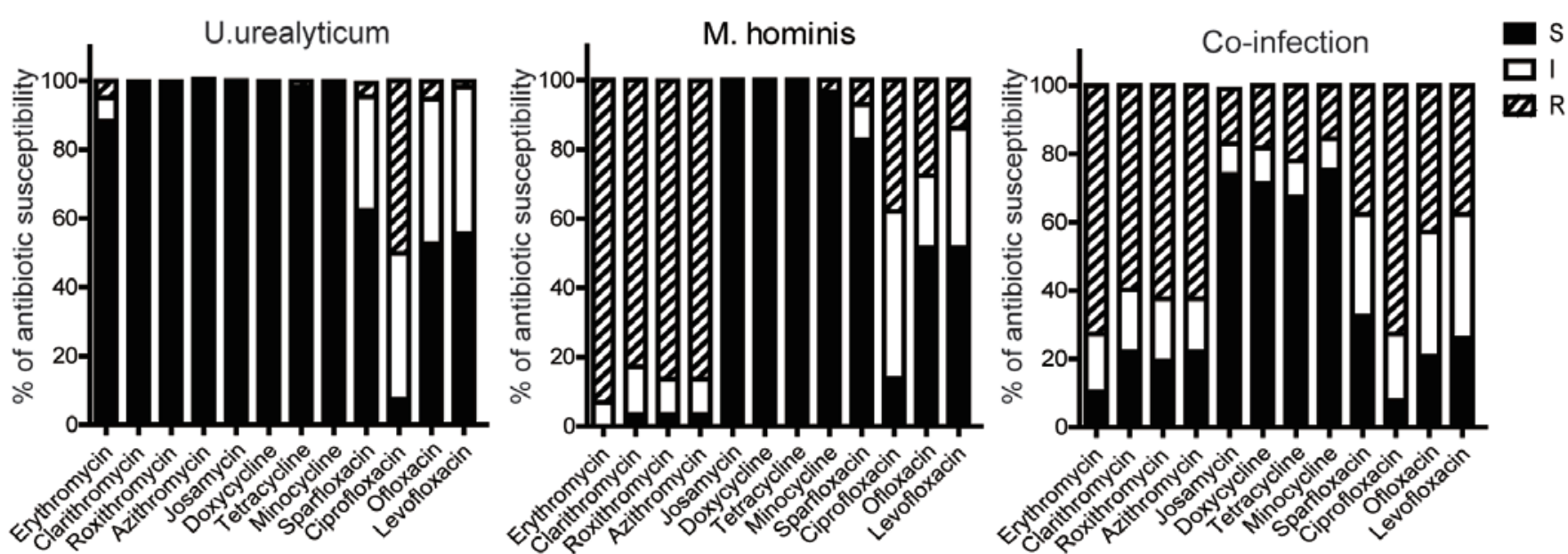

00

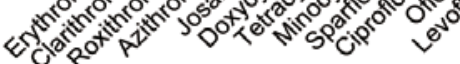

Figure 2. Antimicrobial susceptibility of U. urealyticum, M. hominis and co-infection, respectively. U. urealyticum, Ureaplasma urealyticum; M. hominis, Mycoplasma hominis; S, susceptible; I, intermediate; R, resistant.
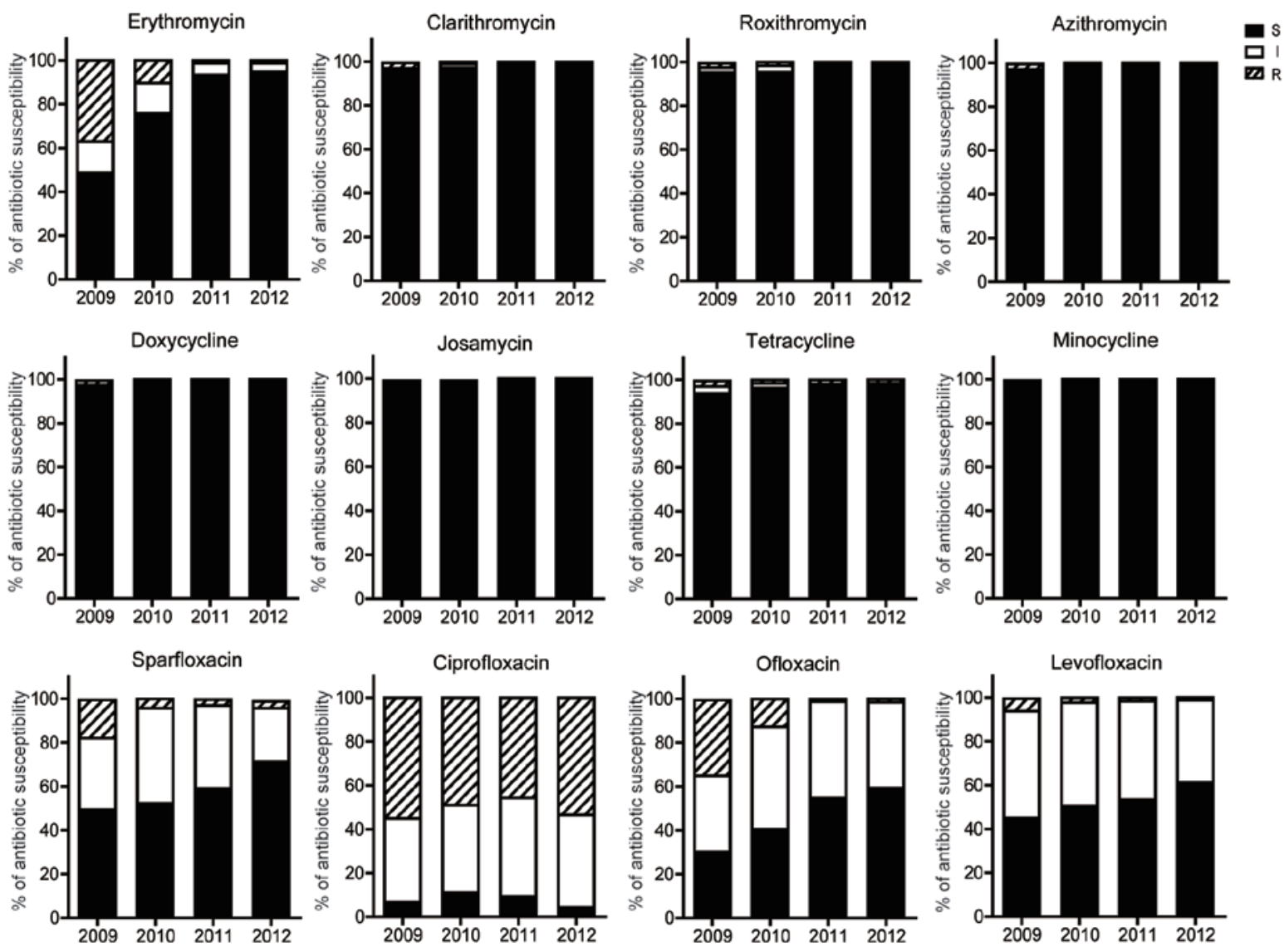

Figure 3. Antimicrobial susceptibility of Ureaplasma urealyticum to different classes of antibiotic over the study period. S, susceptible; I, intermediate; R, resistant.

$M$. hominis co-infection. The susceptibility profiles against both Mycoplasmas over the test period did not change significantly, despite the efficacy of ciprofloxacin to $U$. urealyticum becoming increasingly diminished (Figs. 3 and 4).

Multi-drug resistant (MDR) bacteria have been identified in numerous cases and MDR Mycoplasmas are defined as those strains resistant to at least one agent in $\geq 3$ antimicrobial categories (13). In the present study, the incidence of MDR single $U$. urealyticum infection was significantly reduced, as compared with that of co-infection (1.09 vs. $33.78 \%$; $\mathrm{P}<0.05)$, indicating the presence of cross-resistance in the co-infection patients (Fig. 5).

\section{Discussion}

Worsening environmental contamination and the increasing incidence of sexually transmitted disease, which may lead to infertility, have meant that an increasing number of couples 

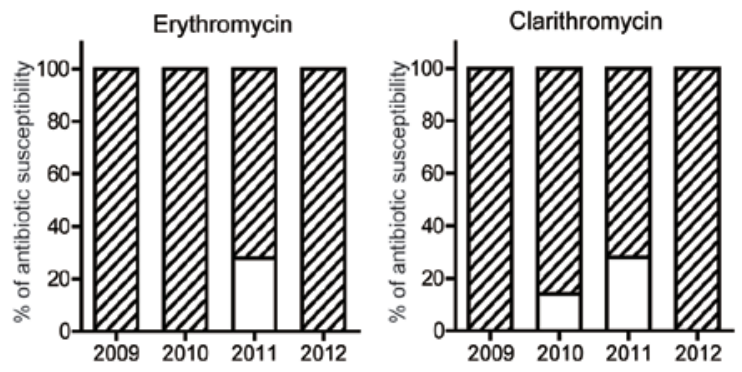

Doxycycline
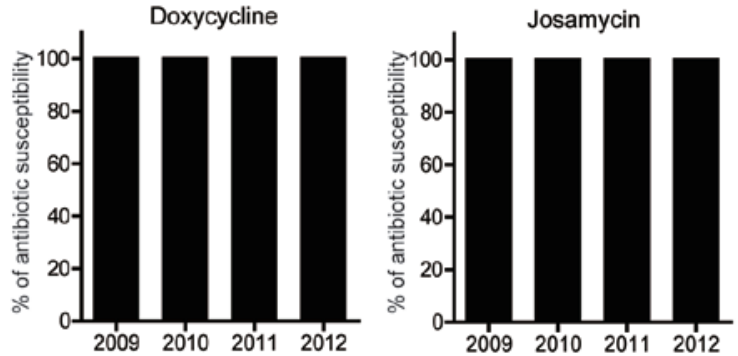

Sparfloxacin
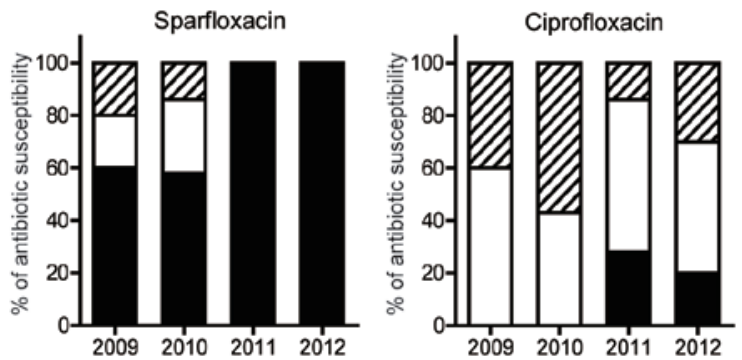
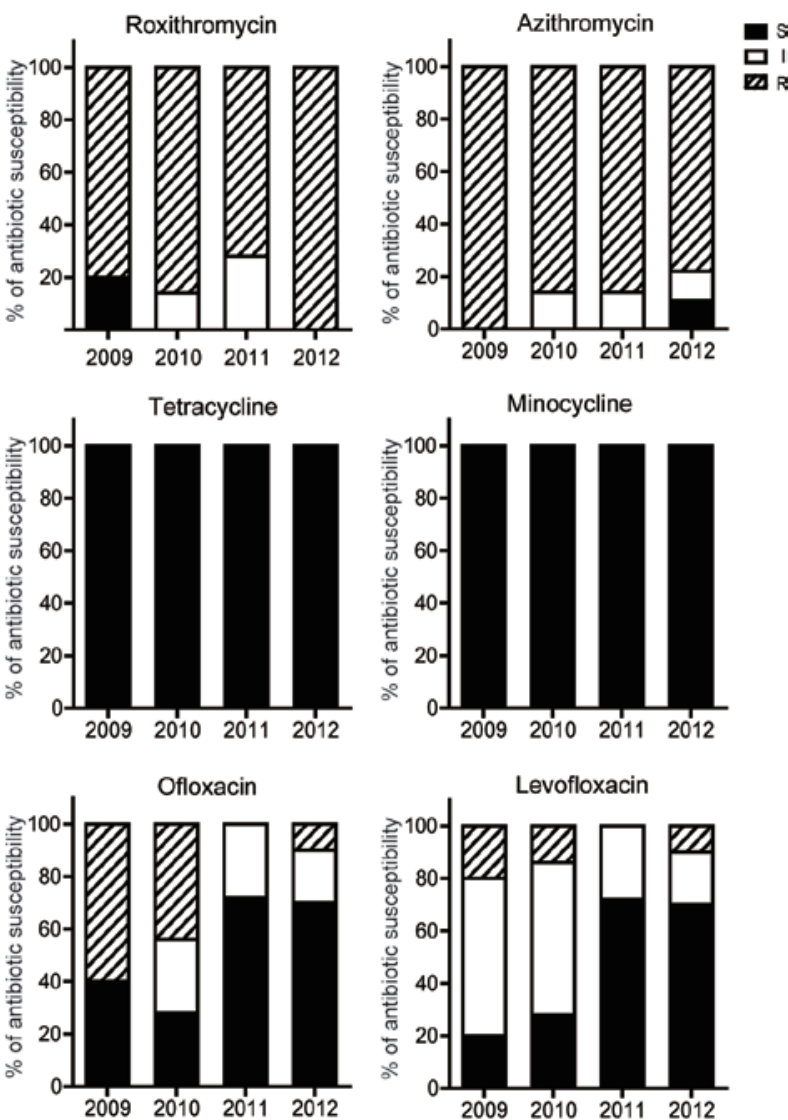

Figure 4. Antimicrobial susceptibility of Mycoplasma hominis to different classes of antibiotic over the study period. S, susceptible; I, intermediate; R, resistant.

will not have their first baby without the aid of assisted reproduction (14). Infertility is emerging as a serious public health issue in China (15). As well as physical and chemical factors, sexually-transmitted infections must also be taken into consideration (16). Mycoplasmas are among the smallest free-living microorganisms. They are commonly isolated from the genitourinary tract of symptomatic patients, but may be found as commensal bacteria from asymptomatic patients. It has been reported that infection with genital Mycoplasmas may lead to pelvic inflammatory disease, puerperal infections, septic abortions, low birth weight, nongonococcal urethritis, prostatitis as well as spontaneous abortion and infertility (17-19). To date, the effect of these microorganisms on male infertility remains unclear, with the exception of limited studies in a few countries $(20,21)$. The present study described the prevalence and antimicrobial susceptibility of $U$. urealyticum and $M$. hominis isolated from semen samples from Chinese patients, and may aid understanding and optimal clinical treatment choice for these pathogens.

This study evaluated differences in the prevalence and antibiotic resistance of $U$. urealyticum and M. hominis. The overall prevalence of infection with either type of bacteria was $43.7 \%$. U. urealyticum was frequently detected as a single pathogen $(42.3 \%)$, and was thus significantly associated with symptomatic patients, including loss of sperm, which indicated its potential pathogenicity. By contrast, a single infection of $M$. hominis $(0.4 \%)$ was rarely found, indicative of disproportionate incidence of these two Mycoplasmas. The present

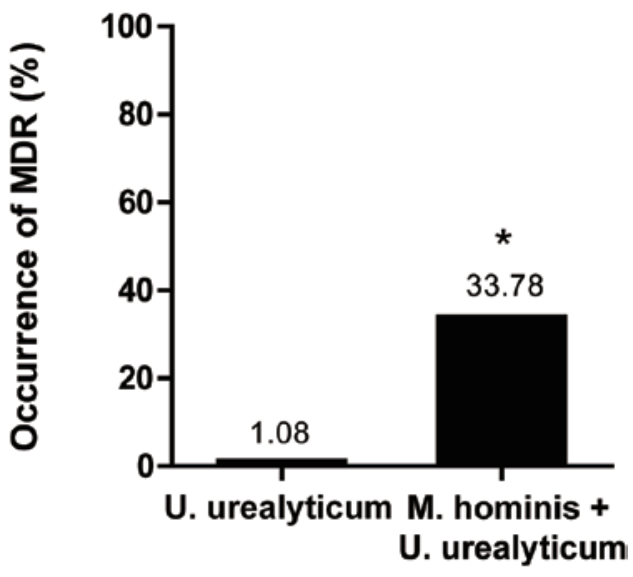

Figure 5. Occurrence of MDR between single U. urealyticum and co-infection specimens. ${ }^{*} \mathrm{P}<0.05$ vs. U. urealyticum only. MDR, multi-drug resistance; U. urealyticum, Ureaplasma urealyticum; M. hominis, Mycoplasma hominis.

findings are consistent with those of other studies performed in Poland (22) and Korea (23); however, they are distinctly different to those of previous studies conducted in Jordan (24) and Italy (25). This discrepancy may be a result of variations in socioeconomic conditions and living standards. In the present study, simultaneous colonization with M. hominis and U. urealyticum was not common $(1.0 \%)$.

In this study, the most frequent occurrence rate of genital Mycoplasma infection was detected in patients aged between 
25 and 34 years old; the age range at which new couples typically conceive their baby. With the exception of the age distribution of the outpatients, no other demographic or clinical characteristics were examined in this study. Other characteristics should be examined in future follow-up studies.

Mycoplasmas are normally susceptible to antibiotics that inhibit protein synthesis, but are resistant to antibiotics that act on bacterial cell wall components because Mycoplasmas do not possess a cell wall (26). The results of this study indicated that there was a difference in sensitivity to the 12 antibiotics between the isolates from single infections and co-infections. Three tetracycline antibiotics (tetracycline, doxycycline and minocycline) and one macrolide antibiotic (josamycin) were active against the majority of the strains. However, four of the quinolone antibiotics (sparfloxacin, levofloxacin, ciprofloxacin and ofloxacin) were inactive against more than one-third of the strains isolated in this study, particularly against the co-infection isolates. Four macrolide antibiotics (azithromycin, erythromycin, clarithromycin and roxithromycin) were effective against the majority of $U$. urealyticum isolates, but were inactive against the majority of bacteria isolates of the single $M$. hominis and co-infections. Consistent with that of female $M$. hominis isolates $(10,11)$, semen isolated $M$. hominis in this study displayed similar resistance spectrum to erythromycin, azythromycin, roxythromycin and clarithromycin. However, a small number of single M. hominis-positive isolates were calculated in the study; thus, further studies including a greater sample size of single M. hominis should be conducted. Although, simultaneous infection with $U$. urealyticum and M. hominis, was not often, but leaded an elevated MDR compared with that of single $M$. hominis infection, indicating a crucial role of cross resistance of these microbes with distinct drug resistant spectrum. The present results support the use of tetracycline, doxycycline, minocycline and josamycin as first choice drugs when empirical therapy is required, whereas the use of erythromycin and quinolones must be carefully considered. The prevalence of the $U$. urealyticum and M. hominis antibiotic resistance profiles in our study were similar to that of female originated Mycoplasmas reported by Wang et al (10) in China, indicating a possible transmission of these Mycoplasmas between men and women. Additionally, no significant difference in antibacterial activity was observed over the study period, with the exception of the reduced activity of ciprofloxacin, which may be attributed to the excessive usage of the drug. Thus, differences in antimicrobial use policies of various areas may also influence the antimicrobial susceptibility characteristics.

In conclusion, the present study retrospectively analyzed the prevalence and antibiotic susceptibility of $U$. urealyticum and M.hominis in semen samples from the Chinese population, in order to provide clinicians with an evidential basis for the rational use of antibiotics, which may be useful for avoiding treatment failure and the abuse of antimicrobial agents.

\section{Acknowledgements}

This study was supported by Research Fund of Jiangsu Provincial Hospital of Traditional Chinese Medicine (grant no. Y14011).

\section{References}

1. Horner P, Blee $\mathrm{K}$ and Adams E: Time to manage Mycoplasma genitalium as an STI: But not with azithromycin $1 \mathrm{~g}$ ! Curr Opin Infect Dis 27: 68-74, 2014.

2. Citti C and Blanchard A: Mycoplasmas and their host: Emerging and re-emerging minimal pathogens. Trends Microbiol 21: 196-203, 2013

3. Waites KB, Katz B and Schelonka RL: Mycoplasmas and ureaplasmas as neonatal pathogens. Clin Microbiol Rev 18: 757-789, 2005.

4. McCormack WM: Susceptibility of mycoplasmas to antimicrobial agents: Clinical implications. Clin Infect Dis 17 (Suppl 1): S200-S201, 1993.

5. Furneri PM, Rappazzo G, Musumarra MP, Di Pietro P, Catania LS and Roccasalva LS: Two new point mutations at A2062 associated with resistance to 16-membered macrolide antibiotics in mutant strains of Mycoplasma hominis. Antimicrob Agents Chemother 45: 2958-2960, 2001.

6. Dégrange S, Renaudin $\mathrm{H}$, Charron A, Bébéar $\mathrm{C}$ and Bébéar CM: Tetracycline resistance in Ureaplasma spp. and Mycoplasma hominis: Prevalence in Bordeaux, France, from 1999 to 2002 and description of two tet (M)-positive isolates of M. hominis susceptible to tetracyclines. Antimicrob Agents Chemother 52: 742-744, 2008.

7. Pereyre S, Renaudin H, Charron A, Bébéar C and Bébéar CM: Emergence of a 23S rRNA mutation in Mycoplasma hominis associated with a loss of the intrinsic resistance to erythromycin and azithromycin. J Antimicrob Chemother 57: 753-756, 2006

8. Mardassi BB, Aissani N, Moalla I, Dhahri D, Dridi A and Mlik B: Evidence for the predominance of a single tet(M) gene sequence type in tetracycline-resistant Ureaplasma parvum and Mycoplasma hominis isolates from Tunisian patients. J Med Microbiol 61: 1254-1261, 2012.

9. Waites KB, Crabb DM, Bing X and Duffy LB: In vitro susceptibilities to and bactericidal activities of garenoxacin (BMS-284756) and other antimicrobial agents against human Mycoplasmas and Ureaplasmas. Antimicrob Agents Chemother 47: 161-165, 2003.

10. Wang QY, Li RH, Zheng LQ and Shang XH: Prevalence and antimicrobial susceptibility of Ureaplasma urealyticum and Mycoplasma hominis in female outpatients, 2009-2013. J Microbiol Immunol Infect 2014 [Epub ahead of print].

11. De Francesco MA, Caracciolo S, Bonfanti C and Manca N: Incidence and antibiotic susceptibility of Mycoplasma hominis and Ureaplasma urealyticum isolated in Brescia, Italy, over 7 years. J Infect Chemother 19: 621-627, 2013.

12. Clinical and Laboratory Standards Institute (CLSI): Methods for antimicrobial susceptibility testing for human mycoplasmas; Approved guideline. CLSI Document M43-A. CLSI, Wayne, PA, pp2-5, 2011.

13. Magiorakos AP, Srinivasan A, Carey RB, Carmeli Y, Falagas ME, Giske CG, Harbarth S, Hindler JF, Kahlmeter G, Olsson-Liljequist B, et al: Multidrug-resistant, extensively drug-resistant and pandrug-resistant bacteria: An international expert proposal for interim standard definitions for acquired resistance. Clin Microbiol Infect 18: 268-281, 2012.

14. Wang YX, You L, Zeng Q, Sun Y, Huang YH, Wang C, Wang P, Cao WC, Yang P, Li YF and Lu WQ: Phthalate exposure and human semen quality: Results from an infertility clinic in China. Environ Res 142: 1-9, 2015.

15. Hu W, Chen M, Wu W, Lu J, Zhao D, Pan F, Lu C, Xia Y, Hu L, Chen $\mathrm{D}$, et al: Gene-gene and gene-environment interactions on risk of male infertility: Focus on the metabolites. Environ Int 91: 188-195, 2016.

16. Gimenes F, Souza RP, Bento JC, Teixeira JJ, Maria-Engler SS, Bonini MG and Consolaro ME: Male infertility: A public health issue caused by sexually transmitted pathogens. Nat Rev Urol 11: 672-687, 2014.

17. Crăcea E, Măicănescu-Georgescu M, Constantinescu S and Botez D: Genital Mycoplasmas and Chlamydiae in male infertility. Arch Roum Pathol Exp Microbiol 41: 219-224, 1982.

18. de Barbeyrac B, Bernet-Poggi C, Fébrer F, Renaudin H, Dupon M and Bébéar C: Detection of Mycoplasma pneumoniae and Mycoplasma genitalium in clinical samples by polymerase chain reaction. Clin Infect Dis 17 (Suppl 1): S83-S89, 1993.

19. Oriel JD: Role of genital mycoplasmas in nongonococcal urethritis and prostatitis. Sex Transm Dis 10 (Suppl 4): S263-S270, 1983. 
20. Al-Sweih NA, Al-Fadli AH, Omu AE and Rotimi VO: Prevalence of Chlamydia trachomatis, Mycoplasma hominis, Mycoplasma genitalium and Ureaplasma urealyticum infections and seminal quality in infertile and fertile men in Kuwait. J Androl 33: 1323-1329, 2012.

21. Gdoura R, Kchaou W, Chaari C, Znazen A, Keskes L, Rebai T and Hammami A: Ureaplasma urealyticum, Ureaplasma parvum, Mycoplasma hominis and Mycoplasma genitalium infections and semen quality of infertile men. BMC Infect Dis 7: 129, 2007.

22. FilipiakE,MarchlewskaK,OszukowskaE,Walczak-JedrzejowskaR, Swierczynska-Cieplucha A, Kula K and Slowikowska-Hilczer J: Presence of aerobic micro-organisms and their influence on basic semen parameters in infertile men. Andrologia 47: 826-831, 2015.

23. Lee JS, Kim KT, Lee HS, Yang KM, Seo JT and Choe JH: Concordance of Ureaplasmaurealyticum and Mycoplasma hominis in infertile couples: Impact on semen parameters. Urology 81: 1219-1224, 2013.
24. Abusarah EA, Awwad ZM, Charvalos E and Shehabi AA: Molecular detection of potential sexually transmitted pathogens in semen and urine specimens of infertile and fertile males. Diagn Microbiol Infect Dis 77: 283-286, 2013.

25. Salmeri M, Valenti D, La Vignera S, Bellanca S, Morello A, Toscano MA, Mastrojeni S and Calogero AE: Prevalence of Ureaplasma urealyticum and Mycoplasma hominis infection in unselected infertile men. J Chemother 24: 81-86, 2012.

26. Taylor-Robinson D and Bébéar C: Antibiotic susceptibilities of Mycoplasmas and treatment of mycoplasmal infections. J Antimicrob Chemother 40: 622-630, 1997. 